\title{
Phase Transitions in Two Dimensions: The Case of Sn Adsorbed on Ge(111) Surfaces
}

\author{
O. Bunk,* J. H. Zeysing, G. Falkenberg, and R. L. Johnson \\ II. Institut für Experimentalphysik, Universität Hamburg, Luruper Chaussee 149, D-22761 Hamburg, Germany
}

M. Nielsen, M. M. Nielsen, and R. Feidenhans'1

Condensed Matter Physics and Chemistry Department, Ris $\phi$ National Laboratory, DK-4000 Roskilde, Denmark (Received 9 March 1999)

\begin{abstract}
Accurate atomic coordinates of the room-temperature $(\sqrt{3} \times \sqrt{3}) R 30^{\circ}$ and low-temperature $(3 \times 3)$ phases of $1 / 3$ monolayer $\mathrm{Sn}$ on $\mathrm{Ge}(111)$ have been established by grazing-incidence $\mathrm{X}$-ray diffraction with synchrotron radiation. The $\mathrm{Sn}$ atoms are located solely at $T_{4}$ sites in the $(\sqrt{3} \times \sqrt{3}) R 30^{\circ}$ structure. In the low-temperature phase one of the three $\mathrm{Sn}$ atoms per $(3 \times 3)$ unit cell is displaced outwards by $0.26 \pm 0.04 \AA$ relative to the other two. This displacement is accompanied by an increase in the first to second double-layer spacing in the Ge substrate.

PACS numbers: 68.35.Bs, 68.35.Rh
\end{abstract}

Phase transitions at surfaces have aroused considerable interest among both theoreticians and experimentalists because they impact a wide variety of fields ranging from industrially important catalytic processes to providing insights into phenomena observed in cuprate superconductors. The suggestion that a commensurate charge density wave can form in $\mathrm{Pb}[1]$ and $\mathrm{Sn}$ [2,3] overlayers on $\mathrm{Ge}(111)$ demonstrated the importance of such simple model systems as testing grounds for modern theories [4]. Upon cooling, both of these adsorbate systems undergo a structural phase transition from a surface reconstruction with a $(\sqrt{3} \times \sqrt{3}) R 30^{\circ}$ periodicity at room temperature to a $(3 \times 3)$ periodicity at low temperatures. For the $\mathrm{Pb} / \mathrm{Ge}(111)$ system the $(3 \times 3)$ structure is accompanied by a small gap opening up in the electronic band structure, indicative of a metal-insulator transition. The picture of a symmetry breaking transition was seriously questioned in two recent papers, which reported almost identical electronic structures for both phases $[5,6]$. The transition was proposed to be of order/disorder type with the Sn atoms fluctuating between two positions at room temperature, but freezing into an ordered $(3 \times 3)$ structure at low temperature with an outwards displacement of every third Sn atom [6]. This throws into question the generally accepted $T_{4}$ model for the $(\sqrt{3} \times \sqrt{3}) R 30^{\circ}$ structure, in which the adsorbate atom is located at a single threefold hollow site above a second layer Ge atom [7] as shown in Fig. 1. To add to the confusion the postulated Sn atom displacement in the $(3 \times 3)$ structure was not found in a recent surface $\mathrm{x}$-ray diffraction (SXRD) study [8].

Challenged by the discrepancies between the electronic and structural studies performed so far, we decided to undertake a thorough investigation using surface $\mathrm{x}$-ray diffraction to determine the geometrical structure of the $\mathrm{Ge}(111)-\mathrm{Sn}$ system both at room and low temperature and by comparison to determine unambiguously the nature of the phase transition.

The samples were prepared in an ultrahigh vacuum (UHV) system equipped with reflection high energy electron diffraction, low energy electron diffraction, and a scanning tunneling microscope (STM). The substrates were cleaned using the standard procedure of repeated sputter-anneal cycles $\left(500 \mathrm{eV} \mathrm{Ar}^{+}\right.$ions, $\left.450{ }^{\circ} \mathrm{C}\right)$ until good $c(2 \times 8)$ diffraction patterns were observed. Tin was deposited from a calibrated effusion cell with the $\mathrm{Ge}(111)$ substrate held at room temperature; afterwards the sample was annealed to $\sim 150^{\circ} \mathrm{C}$. This procedure yielded a $\operatorname{Ge}(111)-(\sqrt{3} \times \sqrt{3}) R 30^{\circ}$-Sn reconstruction with a tin coverage very close to the ideal value of $1 / 3$ monolayer (ML). STM measurements revealed wellordered domains extending over $\sim 400-600 \AA$ with a typical defect density of $\sim 4 \%$, and the absence of the low coverage "mosaic" phase with a mixture of Sn and Ge adatoms. The sample was then transferred in a portable UHV chamber equipped with a closed-cycle sample cooling system to the BW2 wiggler beam line at HASYLAB for the X-ray diffraction measurements. The X-ray photon energy was set to $8.8 \mathrm{keV}$ and a glancing angle of incidence to $0.8^{\circ}$ was used (i.e., above the critical angle to reduce the uncertainties in the measured intensities arising from mechanical displacements). A data set consisting of 35 symmetry inequivalent in-plane reflections, 250 reflections along 14 fractional order rods, and 62 reflections along three crystal truncation rods (CTRs) was recorded for the $(\sqrt{3} \times \sqrt{3}) R 30^{\circ}$ structure determination. After completing the room-temperature measurements, the sample was cooled until the temperature of the sample holder reached $20 \mathrm{~K}$. For the low-temperature $(3 \times 3)$ phase 278 reflections along 17 fractional order rods and 19 reflections along one CTR were recorded. The three rods specific to the $(3 \times 3)$ structure were rather weak and to optimize the signal to background ratio these were measured with the angle of incidence set to the critical angle and the data were scaled accordingly. The condition of the sample was checked by measuring a standard reflection at hourly intervals. The integrated intensities were corrected for the Lorentz factor, polarization factor, active sample area, and the rod interception appropriate for the $z$-axis geometry 


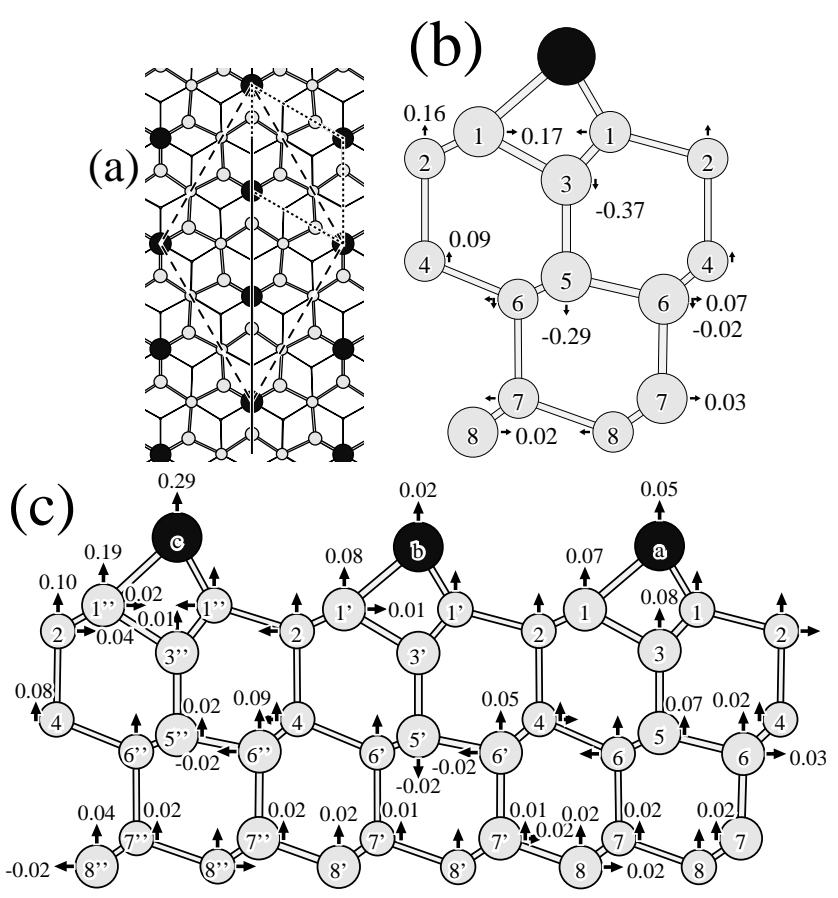

FIG. 1. (a) Top view of the $\mathrm{Ge}(111)-(3 \times 3)-\mathrm{Sn}$ reconstruction. The dashed and dotted lines mark the $(3 \times 3)$ and $(\sqrt{3} \times \sqrt{3}) R 30^{\circ}$ unit cells. The solid line marks the cut for the side views shown in (b) and (c). (b) Side view of the Ge(111)$\sqrt{3} \times \sqrt{3}) R 30^{\circ}-\mathrm{Sn}$ reconstruction. The displacements relative to bulklike positions in [1ํㅣㄹ and [001] directions are given in $\AA$. (c) Side view of the Ge(111)- $(3 \times 3)-S n$ reconstruction. Displacements relative to the room temperature phase shown in (b) are given in $\AA$. For clarity the displacements of symmetry equivalent atoms are shown only once. The atoms are marked with the same labels as in Table I.

[9]. The width of the fractional order reflections from the $(\sqrt{3} \times \sqrt{3}) R 30^{\circ}$ phase corresponded to domains about $500 \AA$ in diameter, and this value did not change upon cooling. The reflections specific to the $(3 \times 3)$ structure were considerably broader corresponding to an average domain size of only $\sim 120 \AA$. This indicates that cooling does not change the basic structure of the surface reconstruction, but it is modified by the superposition of a less well-correlated distortion. In the following we use the conventional surface coordinate system with $\mathbf{a}=$ $\frac{1}{2}[10 \overline{1}]_{\text {cubic }}, \quad \mathbf{b}=\frac{1}{2}[\overline{1} 10]_{\text {cubic }}$, and $\mathbf{c}=\frac{1}{3}[111]_{\text {cubic }}$. The cubic coordinates are in units of the germanium lattice constant (5.66 $\AA$ at $300 \mathrm{~K})$.

A subset of the measured surface diffraction data is shown in Fig. 2. The rods for the $(\sqrt{3} \times \sqrt{3}) R 30^{\circ}$ and the $(3 \times 3)$ phase are very similar, but a careful inspection shows that there are important differences. Some of the rods are basically identical, as can be seen for the $(2 / 3,5 / 3)$ or $(4 / 3,1 / 3)$ rods, whereas for the $(2 / 3,8 / 3)$ or $(7 / 3,1 / 3)$ rods the intensities from the $(3 \times 3)$ structure are significantly higher than those of the $(\sqrt{3} \times \sqrt{3}) R 30^{\circ}$ structure. These differences are due to solely the changes in the atomic positions as a function of temperature.

In order to pinpoint the differences we first determined the atomic positions of the $(\sqrt{3} \times \sqrt{3}) R 30^{\circ}$ structure

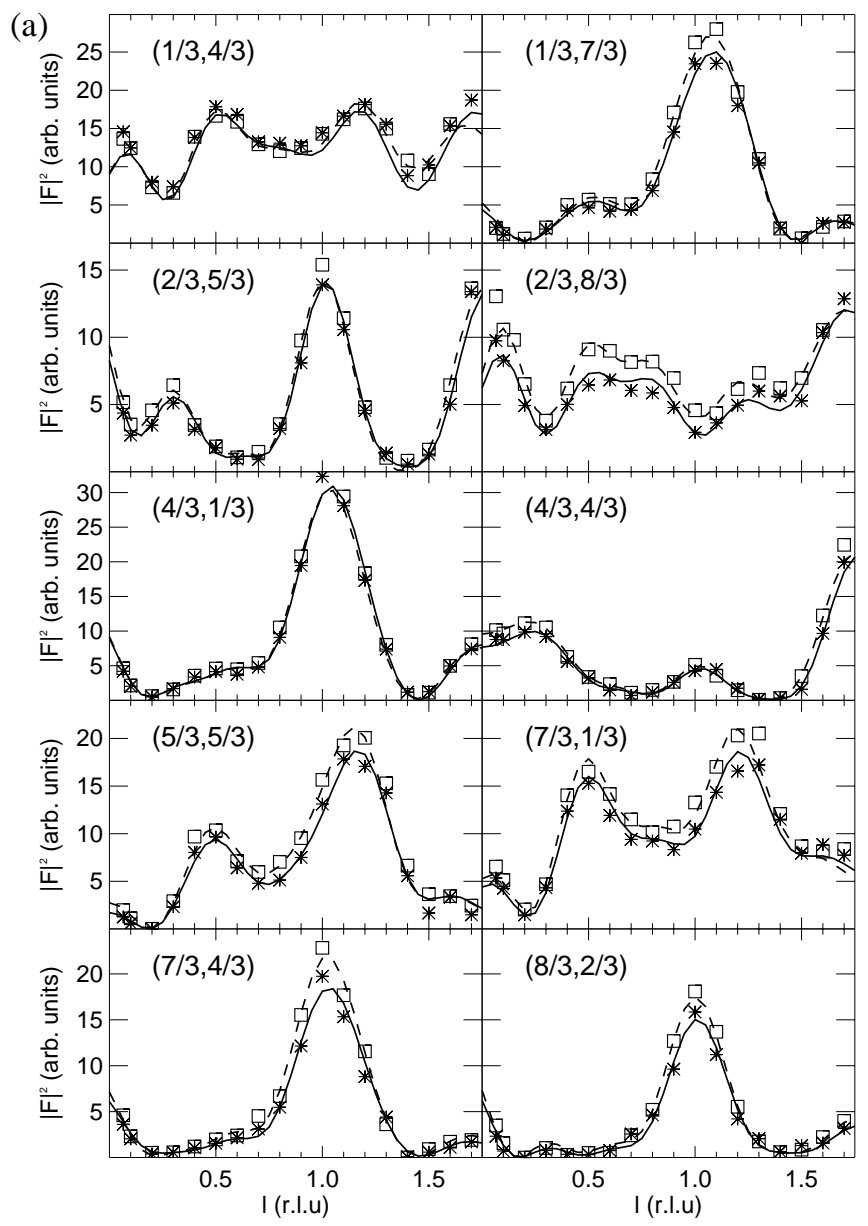

(b)

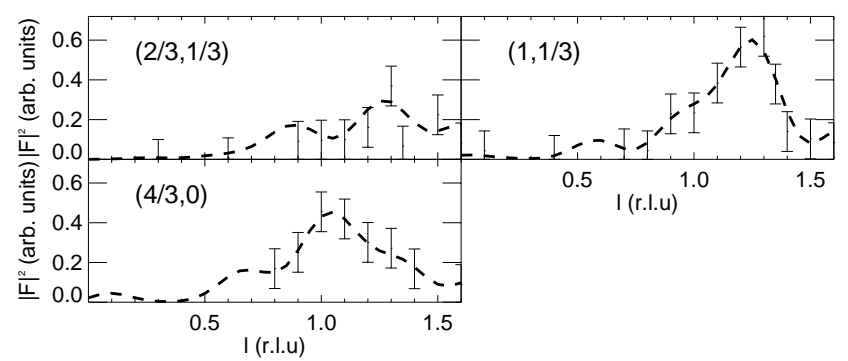

FIG. 2. (a) Comparison between the fractional order rods from both phases. The solid lines are calculated using the parameters for the room temperature phase and the data points for this phase are marked by asterisks. The corresponding curves for the low-temperature phase are indicated by dashed lines and squares. Error bars have been omitted for clarity. Close inspection reveals clear differences in intensity for some rods [e.g., $(2 / 3,8 / 3, l)]$, whereas other rods are nearly identical for both phases [viz. $(4 / 3,1 / 3, l)]$. This proves that the rods specific to the smaller room temperature unit cell are very sensitive to the structural changes upon cooling. (b) Fractional order rods unique to the $(3 \times 3)$ surface reconstruction.

using a least-squares refinement procedure. The atomic coordinates are given in Table I and a ball and stick model of the structure with the displacements relative to bulklike positions is shown in Fig. 1b. The Ge-Ge bond lengths deviate less than $3 \%$ from the bulk value of $2.45 \AA$. The Ge-Sn bonds with $2.83 \pm 0.02 \AA$ are slightly larger than 
TABLE I. The atomic positions (pos.) in the room temperature Ge(111)- $(\sqrt{3} \times \sqrt{3}) R 30^{\circ}$-Sn and low-temperature $(3 \times 3)$ phases and the deviations from ideal bulklike positions in $\AA$. The labels refer to Fig. 1. For symmetry equivalent atoms only one position is given. The estimated uncertainty of the coordinates is about $0.02 \AA$. Isotropic atomic displacement parameters with amplitudes of $0.14 \AA(\mathrm{Sn}), 0.12 \AA$ (four nearest-neighbor $\mathrm{Ge}$ atoms) and $0.09 \AA(\mathrm{Ge})$ were determined for the room temperature phase and amplitudes of $0.41 \AA$ (Sn atom " $c$ " in $z$ direction), $0.04 \AA$ (Sn " $c$ " in-plane, " $a$ " and " $b$ "), $0.15 \AA$ (nearest-neighbor Ge atoms " 1 " " and " 3 "'"), $0.08 \AA$ (remaining Ge atoms in the layers 1-6), and $0.02 \AA$ (bulk Ge) for the low-temperature phase.

\begin{tabular}{|c|c|c|c|}
\hline Atom & Pos. $\sqrt{3}$ & Pos. $(3 \times 3)$ & Dev. $[\AA]$ \\
\hline $\mathrm{a}$ & $(0.333,0.667,0.563)$ & $(0.333,0.667,0.579)$ & \\
\hline $\mathrm{b}$ & & $(2.333,1.667,0.569)$ & \\
\hline $\mathrm{c}$ & & $(1.333,2.667,0.653)$ & \\
\hline 1 & $(0.024,0.048,-0.003)$ & $(0.023,0.047,0.020)$ & $0.17 / 0.17$ \\
\hline $1^{\prime}$ & & $(2.026,1.052,0.022)$ & 0.19 \\
\hline $1^{\prime \prime}$ & & $(1.027,2.054,0.056)$ & 0.26 \\
\hline 2 & $(-0.667,0.667,-0.202)$ & $(2.322,0.657,-0.171)$ & $0.16 / 0.26$ \\
\hline 3 & $(0.333,0.667,-0.364)$ & $(0.333,0.667,-0.341)$ & $0.37 / 0.30$ \\
\hline $3^{\prime}$ & & $(2.333,1.667,-0.364)$ & 0.37 \\
\hline $3^{\prime \prime}$ & & $(1.333,2.667,-0.361)$ & 0.36 \\
\hline 4 & $(-0.667,0.667,-0.971)$ & $(2.334,0.663,-0.948)$ & $0.09 / 0.17$ \\
\hline 5 & $(0.333,0.667,-1.090)$ & $(0.333,0.667,-1.069)$ & $0.29 / 0.23$ \\
\hline $5^{\prime}$ & & $(2.333,1.667,-1.095)$ & 0.31 \\
\hline $5^{\prime \prime}$ & & $(1.333,2.667,-1.084)$ & 0.28 \\
\hline 6 & $(0.677,1.353,-1.257)$ & $(0.681,1.362,-1.251)$ & $0.07 / 0.10$ \\
\hline $6^{\prime}$ & & $(2.675,2.349,-1.240)$ & 0.06 \\
\hline $6^{\prime \prime}$ & & $(1.674,0.349,-1.230)$ & 0.08 \\
\hline 7 & $(0.671,1.343,-2.000)$ & $(0.671,1.341,-1.995)$ & $0.03 / 0.03$ \\
\hline $7^{\prime}$ & & $(2.673,1.327,-1.996)$ & 0.05 \\
\hline $7^{\prime \prime}$ & & $(1.671,0.342,-1.993)$ & 0.04 \\
\hline 8 & $(0.003,0.006,-2.253)$ & $(0.006,0.012,-2.247)$ & $0.02 / 0.04$ \\
\hline $8^{\prime}$ & & $(2.004,1.008,-2.245)$ & 0.03 \\
\hline $8^{\prime \prime}$ & & $(1.000,0.000,-2.241)$ & 0.03 \\
\hline
\end{tabular}

the sum of the tetrahedral covalent radii for germanium and white tin $(2.74 \AA / 2.82 \AA)$ and larger than the value expected for grey tin $(\alpha-\mathrm{Sn})$ and germanium $(2.63 \AA)$. The $\mathrm{Sn}$ bond angle is $82.0^{\circ}$. The in-plane displacement of the first layer Ge atoms of $0.17 \AA$ is significantly larger than the value of $0.05 \AA$ given in Ref. [8] and indicates that the earlier analysis was based on a too limited dataset. The results of a Keating energy minimization are incompatible with the smaller value, so we are forced to conclude that the analysis presented in Ref. [8] is incorrect. As shown in Fig. 2 the curves calculated using our structural model reproduce the experimental data extremely well, and this is confirmed by the reduced $\chi^{2}$ value of 1.6.

Next, we determined the atomic coordinates of the low-temperature $(3 \times 3)$ reconstruction and obtained the values listed in Table I. The differences between the $(3 \times$ $3)$ structure and the $(\sqrt{3} \times \sqrt{3}) R 30^{\circ}$ structure are illustrated in Fig. 1c. There are several important features to be noted: (i) One $\mathrm{Sn}$ atom is displaced out of the surface plane by $0.29 \AA$; this $\mathrm{Sn}$ atom is at a vertical position $0.26 \AA$ higher than the average position of the two lower Sn atoms. (ii) The three nearest-neighbor Ge atoms partially follow this relaxation, mainly in the $z$ direction and not in-plane, contrary to what was reported in Ref. [8]. (iii) The average layer spacing between the first and second Ge double layer is expanded relative to the room-temperature phase.
For the room-temperature phase this distance is 1.004 and from the second to the third double layer 0.993 in lattice coordinates, i.e., an expansion and a contraction relative to the bulk value of 1.000. However, for the low-temperature phase the first to second double-layer distance is 1.026 and the second to third layer distance is 1.002 , i.e., a considerable expansion in the upper two double layers. (iv) The outwards displaced Sn atom has a very anisotropic atomic displacement parameter (adp) with an amplitude 10 times larger in the $z$ direction than in-plane. This means that either the atom is performing a very anisotropic motion with a large amplitude or, as is more likely at low temperatures, there is some disorder in the $z$ position of this atom. The adp's for the nearest-neighbor Ge atoms are also larger than at room temperature, again indicative of disorder. This is not surprising since the position of these atoms must at least partially follow the $\mathrm{Sn}$ atoms. The reduced $\chi^{2}$ for the low-temperature data is 1.3 ; a subset of the fractional order rods is shown in Fig. 2.

A trial using a single isotropic adp for all $\mathrm{Sn}$ atoms resulted in an increased outward displacement of one $\mathrm{Sn}$ atom and an inward displacement of the two other Sn atoms with a total height difference of $\sim 0.45 \AA$ between the Sn atoms. However, the three rods specific to the $(3 \times 3)$ structure were not adequately described by this model.

Several tests were performed to ensure that the features of the low-temperature phase were real and not caused by 
artifacts or local minima in the $\chi^{2}$ minimization. First, to check the sensitivity of the structure determination to changes in the relative weight of reflections we set the error bars on all measured reflections equal and reoptimized every parameter. Although there were some minor differences the main features of the outward displacement and highly anisotropic adp for one Sn atom remained. In the second test we optimized the Ge positions in the third to sixth layers using a Keating model to minimize the elastic strain energy [10]. All deviations were less than $0.06 \AA$, so we can rule out the possibility that the good agreement between the measured and calculated intensities arises from unphysical atomic displacements in the substrate. In the third test we checked whether the low-temperature displacements are dependent on the weak rods specific to the $(3 \times 3)$ periodicity, which have larger relative uncertainties than the other rods. By excluding these rods from the data analysis and reoptimizing the parameters only minor changes, typically $<0.03 \AA$, occurred. From these checks we are convinced that our data analysis has revealed the intrinsic features of the low-temperature $(3 \times 3)$ phase.

Now we can address the classification of the transition between the $(\sqrt{3} \times \sqrt{3}) R 30^{\circ}$ and the $(3 \times 3)$ phase in more detail. Recently, it was proposed to be an order/disorder transition $[5,6]$. This would require two different sites for the Sn atom with a height difference of about $0.26 \AA$ even at room temperature. However, if this were the case, there would be no difference between the $(\sqrt{3} \times \sqrt{3}) R 30^{\circ}$ specific rods in the $(\sqrt{3} \times \sqrt{3}) R 30^{\circ}$ and $(3 \times 3)$ phase apart from the thermal motion effects affecting all rods, in contrast to what we observed experimentally as shown in Fig. 2. To quantify this, we used the $(3 \times 3)$ low-temperature structure and optimized the displacements using the room-temperature data. This gave a more isotropic adp for the outwards displaced $\mathrm{Sn}$ atom and a reduction of the outwards displacement to $0.07 \AA$. The reduced $\chi^{2}$ in this test increased compared to the best fit for the $(\sqrt{3} \times \sqrt{3}) R 30^{\circ}$ structure from 1.6 to 1.7 due to the increase in the number of free parameters. Hence, we can conclude that if there is more than one site for the $\mathrm{Sn}$ atoms in the $(\sqrt{3} \times \sqrt{3}) R 30^{\circ}$ structure the height difference is much less than that observed in the $(3 \times 3)$ phase. The fact that the adp of the surface layer Ge atoms are similar in both phases is strong evidence against an order/disorder phase transition. At low temperatures one would normally expect both reduced thermal motion and disorder. The experimentally observed lattice distortion is reminiscent of a pseudo-Jahn-Teller effect [11] in which the energy of the system is lowered by a spontaneous symmetry-reducing displacement.

In summary, by performing a detailed analysis of comprehensive sets of $\mathrm{x}$-ray diffraction data we have established definitive structural models for both the room-temperature $\mathrm{Ge}(111)-(\sqrt{3} \times \sqrt{3}) R 30^{\circ}$-Sn and lowtemperature $\mathrm{Ge}(111)-(3 \times 3)-\mathrm{Sn}$ surface reconstructions. The atomic coordinates are given in Table I. The major feature of the $(3 \times 3)$ structure is the outward displace- ment of one Sn atom by $0.26 \pm 0.04 \AA$ with respect to the average position of the other two $\mathrm{Sn}$ atoms per unit cell. The three nearest-neighbor $\mathrm{Ge}$ atoms bonding to the displaced $\mathrm{Sn}$ atom are also displaced outwards. In addition there is an increase in the average layer spacing between the first to second Ge double layers compared to the $(\sqrt{3} \times \sqrt{3}) R 30^{\circ}$ phase. We have shown that the phase transition from the $(\sqrt{3} \times \sqrt{3}) R 30^{\circ}$ to the $(3 \times 3)$ phase is not an order/disorder transition. We hope that the detailed structural information presented here will provide the foundation for a better theoretical understanding of this interesting model system.

We thank the staff of HASYLAB for their technical assistance. Financial support from the Danish Research Council through Dansync, the Bundesministerium für Bildung, Wissenschaft, Forschung und Technologie (BMBF) under Project No. 05 SB8 GUA6 and the Volkswagen Stiftung is gratefully acknowledged.

Note added. - Zhang et al. have reanalyzed their previously published SXRD data for the Ge(111)/Sn phases [8] in conjunction with IV-LEED data [12] and find an outward displacement of $0.37 \AA$ for one Sn atom. However, the displacements in the substrate differ from the values reported in this Letter. A comparable outward displacement of a Pb atom by $\sim 0.4 \AA$ was described in a recent publication on the $\mathrm{Ge}(111)-(3 \times 3)-\mathrm{Pb}$ structure by Mascaraque et al. [13].

*Email address: Oliver.Bunk@desy.de

[1] J. M. Carpinelli, H.H. Weitering, E.W. Plummer, and R. Stumpf, Nature (London) 381, 398 (1996).

[2] J. M. Carpinelli, H.H. Weitering, M. Bartkowiak, R. Stumpf, and E. W. Plummer, Phys. Rev. Lett. 79, 2859 (1997).

[3] A. Goldoni and S. Modesti, Phys. Rev. Lett. 79, 3266 (1997); 81, 3553(E) (1998).

[4] S. Scandalo, F. Ancilotto, G. L. Chiarotti, G. Santoro, S. Serra, and E. Tosatti, Surf. Sci. 402-404, 808 (1998).

[5] R. I. G. Uhrberg and T. Balasubramanian, Phys. Rev. Lett. 81, 2108 (1998).

[6] J. Avila, A. Mascaraque, E. G. Michel, M. C. Asensio, G. LeLay, J. Ortega, R. Pérez, and F. Flores, Phys. Rev. Lett. 82, 442 (1999).

[7] J. S. Pedersen, R. Feidenhans'1, M. Nielsen, K. Kjær, F. Grey, and R. L. Johnson, Surf. Sci. 189/190, 1047 (1987).

[8] A. P. Baddorf, V. Jahns, J. Zhang, J. M. Carpinelli, and E. W. Plummer, Phys. Rev. B 57, 4579 (1998).

[9] E. Vlieg, J. Appl. Crystallogr. 30, 532 (1997).

[10] P. N. Keating, Phys. Rev. 145, 637 (1966); J. S. Pedersen, Surf. Sci. 210, 238 (1989).

[11] $\breve{S}$. Pick, Surf. Sci. Rep. 12, 99 (1990).

[12] J. Zhang, Ismail, P. J. Rous, A.P. Baddorf, and E.W. Plummer, Phys. Rev. B 60, 2860 (1999).

[13] A. Mascaraque, J. Avila, J. Alvarez, M.C. Asensio, S. Ferrer, and E. G. Michel, Phys. Rev. Lett. 82, 2524 (1999). 